\title{
Pharmacokinetic Drug Interactions of Azoles
}

\author{
Roger J.M. Brüggemann, PharmD, Jan-Willem C. Alffenaar, PharmD, \\ Nicole M.A. Blijlevens, $M D, P h D$, Eliane M. Billaud, $M D, P h D$, \\ Jos G.W. Kosterink, PharmD, PhD, Paul E. Verweij, MD, PhD, \\ and David M. Burger, PharmD, PhD
}

\section{Corresponding author}

Roger J.M. Brüggemann, PharmD

Department of Clinical Pharmacy, Radboud University Nijmegen Medical Centre, PO Box 9101, 6500 HB Nijmegen, The Netherlands. E-mail: R.Bruggemann@akf.umcn.nl

Current Fungal Infection Reports 2008, 2:20-27

Current Medicine Group LLC ISSN 1936-3761

Copyright (C) 2008 by Current Medicine Group LLC

Drug-drug interactions are a major concern with triazoles. Azoles are potent metabolic inhibitors and interactions commonly occur via metabolizing enzymes (ie, cytochrome P450 isoenzyme superfamily) or drug transporters (ie, P-glycoprotein). However, the clinical relevance of these interactions may vary upon the azole involved and upon the "target" drug. Azoles may also be under influence of and become targets of metabolic drug-drug interactions. Potential interactions between antifungal agents and over-the-counter or alternative medicines and herbs should not be underestimated. Here, we provide a comprehensive overview of different types of pharmacokinetic drug interactions involving azoles with selected examples.

\section{Introduction}

Antifungal drugs have a high potential for drug-drug interactions, especially azoles, which exhibit a wide range and variety of drug-drug interactions. This is an ongoing concern in the treatment of fungal infections. Azoles are well known to inhibit different cytochrome P450 (CYP450) enzyme systems, with CYP3A4 being the major enzyme involved, but are also a substrate to these enzymes. They also are inhibitors of membrane transporters, such as P-glycoprotein (P-gp). Inhibition or induction of CYP450 enzymes may alter the pharmacokinetic profile of the drugs involved and can affect both interacting members and, in most cases, must be avoided as this can lead to overdosing with the consequence of toxicity or underdosing with a loss of effectiveness. The risk of azole interactions with other drug classes can differ substantially between individual drugs, even within the same class of drugs.

In general, it is almost impossible for the clinician to be aware of all interaction mechanisms and potential consequences for the individual patient. Not all interactions are clinically relevant, and a thorough understanding of the mechanisms of drug interaction provides the clinician with the ability to predict such interactions and to avoid those of greatest clinical significance. Regular interaction checks by a clinical pharmacist may aid in solving the problem of drug interactions. Moreover, the avoidance of drug-drug interactions should be regarded as a multidisciplinary task. Now, software tools are available facilitating rapid interaction checks and providing information on the mechanism and clinical relevance of an interaction assisting in the clinical decision making.

This article reviews a subset of pharmacokinetic drug interactions of azoles used in the treatment of systemic fungal infections: fluconazole, itraconazole, voriconazole, and posaconazole. Consequently, despite its high level of metabolic inhibition and drug-drug interactions, ketoconazole is not addressed in this review.

\section{Mechanisms of Drug Interaction}

Drug interactions can be categorized as pharmacokinetic or pharmacodynamic. Pharmacokinetic interactions occur at the level of drug absorption, distribution, metabolism or excretion. Frequently, the CYP450 metabolizing enzyme system and drug transporters, such as P-gp, are involved in these interactions. The results of these interactions can be a decreased or an increased exposure of both interacting drugs, which, in turn, can lead to reduced efficacy or increased toxicity, respectively. Pharmacokinetic drug interactions are manageable using dose adaptation, eventually supported by therapeutic drug monitoring (TDM).

Pharmacodynamic interactions are those where the pharmacological response to a drug is directly altered without a change in pharmacokinetic properties. This 
means that pharmacodynamic interactions are directly related to the desired or undesired drug effects. This can lead to potentiation of effect (including toxicity) in either an additive or synergistic manner, or antagonism. The clinical management of pharmacodynamic drug interactions in our opinion has to be to reevaluate the choice of the coprescribed drug, achieving the best benefit-risk ratio on an individual basis.

Mechanisms involved in pharmacokinetic drug interactions with selected examples are outlined in the following sections.

\section{Drug Absorption}

All four antifungal drugs discussed in this review can be given orally and require absorption through the mucous membranes of the gastrointestinal tract. A change in plasma concentrations can be the result of incomplete drug absorption. Drug absorption of azoles is influenced by acid-lowering drugs (such as $\mathrm{H} 2$-antagonists and proton pump inhibitors); food may exert an effect on the bioavailability of these drugs as well. Pharmacokinetics and bioavailability of fluconazole are not affected by food or acid-lowering drugs.

However, the concomitant administration of itraconazole and proton pump inhibitors or $\mathrm{H} 2$-antagonists leads to impaired absorption, yielding a decrease in exposure [1]. Systemic bioavailability of itraconazole capsules is optimized when taken with food whereas bioavailability of itraconazole oral solution is optimized under fasting conditions [1].

Voriconazole's pharmacokinetic profile is not influenced by antacids or proton pump inhibitors. However, mean maximum plasma concentrations (Cmax) and area under the curve (AUC) of voriconazole were (clinically irrelevant) increased by $15 \%$ by omeprazole due to the inhibition of plasma clearance of voriconazole [2]. Single and multiple oral administration of voriconazole with food lowered the bioavailability by approximately $22 \%$ and delayed absorption compared with a fasting state [3]. Administration of voriconazole with a high-fat meal reduced mean Cmax and AUC by $34 \%$ and $24 \%$, respectively. For this reason, oral dose administration is recommended either 1 hour before or 1 hour after meals [4].

Posaconazole's Cmax and AUC were reduced by $39 \%$ when coadministered with cimetidine $(400 \mathrm{mg}$ twice daily) due to reduced absorption possibly secondary to a decrease in gastric acid production. The effects of other H2-receptor antagonists (eg, famotidine, ranitidine) and proton pump inhibitors (eg, omeprazole) that may suppress gastric acidity for several hours on plasma levels of posaconazole has not been studied but a reduction in bioavailability may occur so that co-administration should be avoided if possible [5]. In the fasting state, antacid slightly increased the relative oral bioavailability of posaconazole by $15 \%$. In the nonfasting state, antacids decreased the relative bioavailability of posaconazole by $12 \%[5,6]$.
The mean increases in posaconazole AUC and Cmax values were about $400 \%$ when administered with a highfat meal compared with the fasting state. Administration of the suspension with a nonfat meal enhanced exposure, resulting in an increase in AUC by $264 \%$ and in Cmax by $296 \%$, relative to the fasting state [6,7]. Coadministration of posaconazole with a nutritional supplement increased the Cmax and AUC 340\% and 260\%, respectively, compared with those for the fasting state. Exposure to posaconazole when given with a nutritional supplement is similar to that when given with a regular meal [8].

The gastric $\mathrm{pH}$ and the gastrointestinal cell wall composition are important in the absorption of antifungal drugs. Posaconazole absorption appeared to be reduced in patients with grade 1 to 2 mucositis compared with that of patients without mucositis (AUC $4.54 \mathrm{mg}$ *h/L vs 8.85 $\mathrm{mg} * \mathrm{~h} / \mathrm{L}$ for twice daily $400 \mathrm{mg}$ posaconazole) [9].

\section{Drug Transporters}

In addition to CYP enzymes, active transporters, such as Pgp, organic anion-transporting polypeptides (OATP), and breast cancer resistant protein (BCRP), play an important role in drug interactions. These active drug transporters regulate the access of drugs to the drug-metabolizing enzymes and control drug concentrations in enterocytes and hepatocytes. A contribution of efflux transporters in drug-drug interactions cannot be excluded $[10,11]$.

Coadministration of azoles with P-gp substrates is known to cause an effect [10]. Two of these azoles, itraconazole and posaconazole, are substrates and inhibitors of the MDR-1 gene product P-gp [5,11]. Much of the data on the effects of the antifungal agents on P-gp comes from in vitro cell culture models of directional drug transport or ex vivo models of intestinal drug secretion. The results obtained seem to vary with the model used, making a definitive conclusion difficult. In vivo, no concrete relations between azoles and P-gp have been established [10].

OATP1B1 is a multispecific carrier capable of bidirectional transport across the sinusoidal liver membrane [12]. This specific enzyme is involved in the transport of different drugs. It is suggested that itraconazole might block transport of atorvastatin due to inhibition of the OATP1B1 enzyme system [13]. However, the exact role of OATP has not been established, and thus, its specific role remains unclear and needs to be investigated.

BRCP belongs to the adenosine triphosphate-binding cassette transporter family. It is expressed in the apical membrane of the epithelium in the small intestine and the liver canalicular membrane. It is highly likely that BCRP is inhibited by itraconazole but is not inhibited by fluconazole and voriconazole [14]. Thus, BCRP could play a significant role in the pharmacokinetic interactions of itraconazole with BCRP substrate drug. The relationship between posaconazole and BRCP has not been investigated. In vivo, no concrete relations between azoles and BCRP have been established. 


\section{Metabolism}

The major site of drug metabolism is the liver, where two types of reactions occur. Metabolism of most drugs occurs via phase 1 reactions (oxidation, reduction, and hydrolysis). The most important enzymes involved in phase 1 reactions are the CYP450 enzymes. In addition, phase 2 reactions are not mediated by CYP enzymes but involve conjugation of the drugs. Drugs can be substrates, inducers, and inhibitors. Substrates are moieties that undergo metabolism by one or more CYP enzymes. Enzyme induction can lead to an increased (in case of the use of a prodrug) and a decreased drug effect whereas enzyme inhibitors decrease the amount of enzyme activity. It may take days up to 2 to 3 weeks, depending on the drug and its dosage, to fully develop enzyme induction. In contrast to induction, enzyme inhibition can occur almost immediately. The effect of the drug on the CYP enzyme is concentration-dependent. The CYP group of enzymes can be highly substrate-specific and, therefore, capable of metabolizing only few substrates. They also may be aspecific and capable of metabolizing a broad range of substrates. The degree of enzyme specificity greatly affects the outcome of its inhibition. The broader the enzyme specificity, the more drug interactions its inhibition is likely to elicit.

\section{Effect of Azoles on Comedicated Drugs}

All azoles are inhibitors of the CYP3A4 isoenzyme. In humans, CYP3A has the largest fraction of the total CYP content and is responsible for the metabolism of a broad spectrum of drugs. For instance, HMG-CoA reductase inhibitors, calcium-channel blockers, and immunosuppressant drugs are known substrates of CYP3A4. Furthermore, CYP3A4 is located in the small bowel and liver and, therefore, is involved in presystemic (first-pass) metabolism, thus influencing the absorption of CYP3A substrates in the gastrointestinal tract. The inhibiting potencies of the azoles are vastly different. Itraconazole is considered a more potent inhibitor of CYP3A4 than posaconazole and voriconazole, which in turn, are more potent than fluconazole. Next to CYP3A4, fluconazole, itraconazole, and voriconazole have inhibiting potencies for various other isoforms, such as CYP2C9 and CYP2C19 [15-17]. The clinical relevance will depend on the CYP isoforms that metabolize the coadministered drug, the intrahepatic concentration of the antifungal agent, and the potency of the antifungal agent as an inhibitor of the isoforms that metabolize the coadministered drug and vice versa.

\section{Oral anticoagulants}

Understandably, drug interactions with oral anticoagulants are always of concern, especially when they are combined with antifungal drugs. Several interactions between azoles and anticoagulants have been reported $[15,18,19]$. There is an increased risk of bleeding in patients taking concomitant azoles and anticoagulants. Therefore, in general, it is recommended to monitor coag- ulation variables (international normalized ratio) when initiating and stopping azole therapy.

\section{Immunosuppressant drugs}

Calcineurin inhibitors (cyclosporine and tacrolimus) and proliferation signal inhibitors (sirolimus and everolimus) are substrates of CYP3A4 and substrates and inhibitors of P-gp. As a consequence, they have immense potential for clinically significant drug interactions that result in increased whole-blood concentrations of the immunosuppressant drug.

Cyclosporine-fluconazole interaction is controversial and may result in a nonclinically relevant increase of concentration of cyclosporine [20,21], whereas concomitant administration of voriconazole and cyclosporine resulted in a 1.7- to 2.5-fold increase of cyclosporine [22]. The effect of itraconazole showed a mean $80 \%$ increase of cyclosporine concentration [23,24]. This interaction effect is based on the intravenous admixtures of itraconazole and cyclosporine. The effect of oral formulations might be more extensively due to inhibition of mucosal CYP enzymes. Posaconazole was the least potent inhibitor of cyclosporine, resulting in an increase of AUC of $30 \%$ (range 20.7-27.3 ng*h/mL). The effect of the interaction may be limited because of the low dose of posaconazole administered (200 mg once daily). It is not known whether higher doses would yield a stronger effect.

Tacrolimus's pharmacokinetic profile is influenced in a similar qualitative-profile way as cyclosporine but exhibits higher intensities. Posaconazole increased the Cmax and AUC for tacrolimus by $121 \%$ and $358 \%$, respectively, on day 14 compared with day 1 [24,25]. A case report described a 3.5-fold increase of tacrolimus when combined with voriconazole [26]. Fluconazole exerts a significant effect on tacrolimus resulting in a twofold increased exposure. Itraconazole represents a potent inhibitor of tacrolimus metabolism varying from twofold in renal transplant patients up to fivefold in lung transplant patients; therefore, this association must be carefully monitored [24,27,28].

Sirolimus's pharmacokinetics are strongly affected by voriconazole [29], and a decrease of $90 \%$ of sirolimus dosage was necessary to avoid toxicity. No randomized studies with fluconazole, itraconazole, or posaconazole and sirolimus have been performed to assess the interaction. In two case reports, there was a significant influence on sirolimus's AUC and Cmax, caused by fluconazole and itraconazole $[30,31]$. An effect of posaconazole is to be expected.

Only one case report has been published on the interaction between everolimus and fluconazole and itraconazole. Azoles were represented by fluconazole in 16 patients and itraconazole in one patient. Data suggested no significant influence from fluconazole, but a potentially large influence from itraconazole, on everolimus [32]. The patient receiving concomitant itraconazole and everolimus exhibited a $74 \%$ decrease in clearance of everolimus. No 


\begin{tabular}{|c|c|c|c|c|}
\hline Enzyme system & Fluconazole & Itraconazole & Posaconazole & Voriconazole \\
\hline \multicolumn{5}{|l|}{ Inhibitor } \\
\hline 2C9 & $++^{*}$ & + & - & ++ \\
\hline $2 \mathrm{C} 19$ & + & - & - & +++ \\
\hline $3 \mathrm{~A} 4$ & ++ & +++ & +++ & ++ \\
\hline P-glycoprotein & - & Yes & Yes & - \\
\hline \multicolumn{5}{|l|}{ Substrate } \\
\hline 2C9 & - & - & - & + \\
\hline $2 \mathrm{C} 19$ & - & - & - & +++ \\
\hline $3 \mathrm{~A} 4$ & - & +++ & - & + \\
\hline P-glycoprotein & - & Yes & Yes & - \\
\hline
\end{tabular}

published data are available for voriconazole or posaconazole, but such interactions are very likely, and special caution is recommended during coprescription.

Based on these results, it is recommended to reduce the dose of cyclosporine and tacrolimus when coadministered with azoles under careful TDM. The combined use of sirolimus with voriconazole must be avoided but fluconazole can be given simultaneously with frequent monitoring of sirolimus whole-blood concentrations. Itraconazole should be used with extreme caution only and with careful TDM. Concomitant administration of potent inhibitors of CYP3A may decrease the clearance of everolimus and increase its blood levels. Frequent monitoring of everolimus is recommended.

\section{Benzodiazepine sedative drugs}

Coadministration of all azoles with midazolam results in significant pharmacokinetic interactions and alterations in the pharmacodynamics of this agent $[33,34,35 \bullet]$. The combination of azoles and midazolam must be avoided, or midazolam dosage must be reduced and patients must have a clinical follow-up when on intravenous therapy. Also other benzodiazepine drugs, such as diazepam, are influenced by fluconazole, itraconazole, and voriconazole $[36,37]$. No data are available for posaconazole but because diazepam is metabolized by CYP2C19, a relevant effect is not to be expected.

\section{Anticancer drugs}

Voriconazole, posaconazole, and itraconazole are known to interfere with vincristine metabolism and increase its neurotoxicity $[38,39 \bullet, 40 \bullet, 41]$. Enhanced toxicity is due to the inhibition of CYP450-mediated metabolism of vincristine, leading to increased plasma levels of the drug. Itraconazole also inhibits the P-gp efflux pump, which is responsible for increasing the excretion of vinca alkaloids from the cell, resulting in high intracellular vincristine levels. Marr et al. [42] found that itraconazole and fluconazole alter metabo- lism of cyclophosphamide in unique fashions, potentially impacting toxicities early after stem cell transplantation. Enhancement of taxane toxicities is also reported with CYP inhibitors and caution should be required with azoles as well [1]. These observations are sufficient to encourage caution with coadministration of azoles and cytochromemetabolized cytotoxic agents because coadministration of azoles might interfere with the curative therapy.

\section{Effect of Comedicated Drugs on Azoles}

Fluconazole is mainly excreted unchanged in urine and, thus, not under influence of induction or inhibition of a metabolic pathway. Interactions influencing the pharmacokinetic profile of fluconazole through this system are not likely. Itraconazole and voriconazole are metabolized by the liver via phase 1 reactions with different isoenzymes involved as the major metabolic pathway. Itraconazole is predominantly metabolized by CYP3A4 and is the only azole with an active metabolite [43,44], whereas voriconazole is substrate to CYP2C19 and to a lesser extent CYP2C9 and CYP3A4. Posaconazole metabolism involves phase 2 reactions [45] (glucuronidation of the drug) (Table 1). Drugs influencing this phase 2 enzyme systems can exert a change in the pharmacokinetic profile of the azole involved leading to an increased or decreased exposure.

Drug inducers, such as phenytoin and rifampicin, may cause a clinically significant alteration in pharmacokinetic behavior of itraconazole, voriconazole, and posaconazole [46-48]. Repeated dose administration of phenytoin decreased the mean steady-state Cmax and AUC of voriconazole by approximately $50 \%$ and $70 \%$, respectively [46]. Increasing the dose of voriconazole from $200 \mathrm{mg}$ to $400 \mathrm{mg}$ twice daily compensated for this effect. The interaction is a two-way interaction because phenytoin's pharmacokinetics are affected by voriconazole with an increase of the mean steady-state Cmax and AUC of phenytoin by approximately $70 \%$ and $80 \%$, respectively [46]. 
Coadministration of posaconazole with phenytoin resulted in $44 \%$ and $52 \%$ decreases in posaconazole Cmax and AUC, respectively [49].

Because coadministration of enzymatic inducers, such as phenytoin and rifampin, significantly reduces exposure of azoles, concomitant use of these agents should be avoided unless the benefit outweighs the risk. Because fluconazole is mainly excreted by the kidneys and not metabolized hepatically, it is not under significant influence of enzyme inducers and, thus, could be considered as a therapeutic option in case of appropriate indication, such as a candidiasis.

Also, HIV protease inhibitors (eg, ritonavir) and nonnucleoside reverse transcriptase inhibitors (eg, efavirenz) cause a clinically significant change in azole exposure $[50,51,52 \bullet, 53,54 \bullet]$. The interaction between voriconazole and efavirenz is considered a two-way interaction because both voriconazole and efavirenz are affected when coadministered [54•]. Enzymatic inhibitors, such as erythromycin and clarithromycin, may play a significant role in drug interactions and should be considered for azole inhibition. Inhibition could enhance the risk of azole toxicities, especially hepatotoxicity [55].

TDM comprises the measurement and interpretation of drug concentrations in biological fluids to assist in the determination of drug dosage for the individual patient. TDM integrates dose, concentration, and effect in order to understand more precisely the pharmacokinetics and pharmacodynamics of the drug in an individual. The risk of subtherapeutic concentrations due to induction of the metabolic turnover of azoles is an indication for TDM. When TDM yields subtherapeutic levels, the dose of the specific drug involved can be increased. This should improve response to therapy, and thus be considered a tool to manage drug-drug interactions and prevent therapeutic failure.

\section{Genetic Polymorphism}

\section{of CYP450 Enzyme Systems}

The main CYP450 enzymes involved in the metabolization of azoles include CYP2C9, CYP2C19, and CYP3A4/3A5. All enzymes are known to have multiple polymorphisms that divide the general population into poor (PM) and extensive metabolizers (EM). Polymorphisms of CYP2C9 and CYP2C19 may play a clinically relevant role in the pharmacokinetics of selected azoles. In homozygous PMs of CYP2C19, the Cmax and AUC of voriconazole are approximately two to five times higher than for EMs [56]. By blocking the CYP3A4 pathway through administration of ritonavir in PM homozygous patients, voriconazole exposure can increase up to $800 \%$ compared with wild-type genotype [56]. The CYP2C9 genotypic variation does not influence the exposure to voriconazole [57] as only a small fraction of the drug is metabolized through this enzymatic pathway. The prevalence of variations in the gene sequence differs by race [58]. Assessing the genotype of patients may predict possible toxicity especially in Asian patients who may be at risk because the frequency of PMs is higher resulting in higher plasma concentrations and toxicity. TDM may be used as a tool to determine high exposure to azoles. When TDM yields high concentrations the genotype may be assessed in order to determine the cause of the high concentration of voriconazole. Determining the patient's genotype before initiation of therapy is not recommended; rather, using clinical signs to determine toxicity together with monitoring of plasma concentrations is more suitable. On the contrary, a drug interaction study can be more valuable if performed with the knowledge of patients' genotypic status.

\section{Excretion}

Drug interactions based on alterations in renal elimination mainly involve changes in tubular secretion or changes in kidney function. Drugs that use the same active transport system in the kidney tubules can compete for this excretory system. The two drugs excreted by the kidneys are fluconazole and hydroxyitraconazole. No interaction reports have been published dealing with impaired renal function caused by nephrotoxic drugs, such as cyclosporine or gentamicin, leading to increased exposure of fluconazole or hydroxyitraconazole and, thus, increased toxicity.

\section{Over-the-counter and Alternative Medicines}

The risks associated with alternative (herbal) medicines, over-the-counter products (eg, vitamins, NSAIDs), and nutritional supplements when used in combination with antifungals are of most concern. Most of these interactions are grossly under-reported. Widespread use of these products and a perceived lack of awareness of risk could confront the patient and their doctor with sudden, unexpected toxic effects or loss of efficacy. Typical examples include the use of St. John's wort and grapefruit juice. Coadministration of grapefruit juice and itraconazole produced a statistically significant, but clinically irrelevant, increase in itraconazole oral solution AUC of $17 \%$. Apparent oral clearance of itraconazole oral solution was significantly reduced $(14 \%)$ which is caused by inhibition of intestinal CYP3A4 [59]. Concurrent use of grapefruit juice and itraconazole capsules resulted in an even stronger effect: a $43 \%$ decrease in the mean itraconazole AUC and a $47 \%$ decrease in the mean hydroxyitraconazole AUC. It can be concluded that concomitant grapefruit juice does not enhance the systemic availability of itraconazole capsules but appears to impair its absorption. Therefore, the use of grapefruit juice will be most likely to yield subtherapeutic concentrations of itraconazole [60]. No recommendations have been made on the concomitant use of fluconazole or voriconazole with grapefruit juice, but an effect, based on the metabolism 
of the drugs, is most likely not to occur or, at least, not be clinically relevant. The effect of grapefruit juice on posaconazole remains to be determined.

St. John's wort had a clinically significant effect on voriconazole exposure. After an initial, clinically irrelevant, increase of voriconazole AUC, there was a significant $59 \%$ decrease in exposure of voriconazole [61]. The use of St. John's wort is, therefore, contraindicated when on voriconazole therapy.

The risk of combined use of alternative or over-thecounter medicines with azoles will be occasional and intermittent, but most of all, intake is unknown to the treating physician, thus complicating the diagnosis of a drug interaction. When considering drug interactions with alternative and over-the-counter medicines, it is not possible to propose a contraindication in the case of a nonregistered substance, and therefore, we are not able to warn patients on possible risks in case of concomitant use of these products with their antifungal treatment.

\section{Conclusions}

Drug interactions can cause many clinical problems. Metabolic inhibitory effects of azoles are very potent, will yield higher exposure to the coadministered drug and may cause great difficulties in establishing a good therapy due to toxicity.

Ideally, all of this knowledge should be available before a new drug enters clinical testing. Although translation of preclinical data to the clinic remains cumbersome and will never be perfect, we should always aim for the maximum attainable therapeutic effect. Therefore, it is important to continue the collection of more mechanistic and theoretical knowledge about drug interactions.

One should also bear in mind that a drug interaction not only occurs upon initiation of therapy but might also become evident when a drug is stopped. Common knowledge is the key factor.

Awareness of the mechanisms involved in these interactions is pivotal in the optimization of treatment of patients requiring antifungal therapy, together with clinical relevance in terms of severity and scientific documentation, will result in a strategy to safely manage coprescriptions and to establish the level of recommendation associated with the drug interaction (contraindicated, not recommended, warning). Addressing drug-drug interactions is a multidisciplinary task with the goals to minimize unwanted side effects in patients and optimize patient care, and TDM should be considered in the optimization of such pharmacokinetic drug-drug interactions.

\section{Disclosures}

No potential conflicts of interest relevant to this article were reported.

\section{References and Recommended Reading}

Papers of particular interest, published recently, have been highlighted as:

- Of importance

$\bullet \quad$ Of major importance

1. College ter Beoordeling van Geneesmiddelen/Medicines Evaluation Board: Trisporal. http://www.cbg-meb.nl/Bijsluiters/h21024.pdf. Accessed December 20, 2007.

2. Wood N, Tan K, Purkins L, et al.: Effect of omeprazole on the steady-state pharmacokinetics of voriconazole. Br J Clin Pharmacol 2003, 56(Suppl 1):56-61.

3. Purkins L, Wood N, Kleinermans D, et al.: Effect of food on the pharmacokinetics of multiple-dose oral voriconazole. Br J Clin Pharmacol 2003, 56(Suppl 1):17-23.

4. European Medicines Agency: Vfend. http://www.emea. europa.eu/humandocs/Humans/EPAR/vfend/vfend.htm. Accessed December 20, 2007.

5. European Medicines Agency: Noxafil. http://www.emea. europa.eu/humandocs/Humans/EPAR/noxafil/noxafil.htm. Accessed December 20, 2007.

6. Courtney R, Radwanski E, Lim J, Laughlin M: Pharmacokinetics of posaconazole coadministered with antacid in fasting or nonfasting healthy men. Antimicrob Agents Chemother 2004, 48:804-808.

7. Courtney R, Wexler D, Radwanski E, et al.: Effect of food on the relative bioavailability of two oral formulations of posaconazole in healthy adults. Br J Clin Pharmacol 2004, 57:218-222.

8. Sansone-Parsons A, Krishna G, Calzetta A, et al.: Effect of a nutritional supplement on posaconazole pharmacokinetics following oral administration to healthy volunteers. Antimicrob Agents Chemother 2006, 50:1881-1883.

9. Gubbins PO, Krishna G, Sansone-Parsons A, et al.: Pharmacokinetics and safety of oral posaconazole in neutropenic stem cell transplant recipients. Antimicrob Agents Chemother 2006, 50:1993-1999.

10. Venkatakrishnan K, Von Moltke LL, Greenblatt DJ: Effects of the antifungal agents on oxidative drug metabolism: clinical relevance. Clin Pharmacokinet 2000, 38:111-180.

11. Wang EJ, Lew K, Casciano CN, et al.: Interaction of common azole antifungals with $\mathbf{P}$ glycoprotein. Antimicrob Agents Chemother 2002, 46:160-165.

12. Li L, Meier PJ, Ballatori N: Oatp2 mediates bidirectional organic solute transport: a role for intracellular glutathione. Mol Pharmacol 2000, 58:335-340.

13. Lennernas H: Clinical pharmacokinetics of atorvastatin. Clin Pharmacokinet 2003, 42:1141-1160.

14. Gupta A, Unadkat JD, Mao Q: Interactions of azole antifungal agents with the human breast cancer resistance protein (BCRP). J Pharm Sci 2007, 96:3226-3235.

15. Black DJ, Kunze KL, Wienkers LC, et al.: Warfarin-fluconazole. II. A metabolically based drug interaction: in vivo studies. Drug Metab Dispos 1996, 24:422-428.

16. Hyland R, Jones BC, Smith DA: Identification of the cytochrome P450 enzymes involved in the N-oxidation of voriconazole. Drug Metab Dispos 2003, 31:540-547.

17. Johnson LB, Kauffman CA: Voriconazole: a new triazole antifungal agent. Clin Infect Dis 2003, 36:630-637.

18. Purkins L, Wood N, Kleinermans D, Nichols D: Voriconazole potentiates warfarin-induced prothrombin time prolongation. Br J Clin Pharmacol 2003, 56(Suppl 1):24-29.

19. Yeh J, Soo SC, Summerton C, Richardson C: Potentiation of action of warfarin by itraconazole. BMJ 1990, 301:669.

20. Canafax DM, Graves NM, Hilligoss DM, et al.: Interaction between cyclosporine and fluconazole in renal allograft recipients. Transplantation 1991, 51:1014-1018.

21. Sud K, Singh B, Krishna VS, et al.: Unpredictable cyclosporin-fluconazole interaction in renal transplant recipients. Nephrol Dial Transplant 1999, 14:1698-1703. 
22. Romero AJ, Le Pogamp P, Nilsson LG, Wood N: Effect of voriconazole on the pharmacokinetics of cyclosporine in renal transplant patients. Clin Pharmacol Ther 2002, 71:226-234.

23. Florea NR, Capitano B, Nightingale CH, et al.: Beneficial pharmacokinetic interaction between cyclosporine and itraconazole in renal transplant recipients. Transplant Proc 2003, 35:2873-2877.

24. Leather H, Boyette RM, Tian L, Wingard JR: Pharmacokinetic evaluation of the drug interaction between intravenous itraconazole and intravenous tacrolimus or intravenous cyclosporin A in allogeneic hematopoietic stem cell transplant recipients. Biol Blood Marrow Transplant 2006, 12:325-334.

25. Sansone-Parsons A, Krishna G, Martinho M, et al.: Effect of oral posaconazole on the pharmacokinetics of cyclosporine and tacrolimus. Pharmacotherapy 2007, 27:825-834.

26. Kuypers DR, Claes K, Evenepoel P, Vanrenterghem Y: Clinically relevant drug interaction between voriconazole and tacrolimus in a primary renal allograft recipient. Transplantation 2006, 81:1750-1752.

27. Manez R, Martin M, Raman D, et al.: Fluconazole therapy in transplant recipients receiving FK506. Transplantation 1994, 57:1521-1523.

28. Shitrit D, Ollech JE, Ollech A, et al.: Itraconazole prophylaxis in lung transplant recipients receiving tacrolimus (FK 506): efficacy and drug interaction. J Heart Lung Transplant 2005, 24:2148-2152.

29. Marty FM, Lowry CM, Cutler CS, et al.: Voriconazole and sirolimus coadministration after allogeneic hematopoietic stem cell transplantation. Biol Blood Marrow Transplant 2006, 12:552-559.

30. Cervelli MJ: Fluconazole-sirolimus drug interaction. Transplantation 2002, 74:1477-1478.

31. Said A, Garnick JJ, Dieterle N, et al.: Sirolimus-itraconazole interaction in a hematopoietic stem cell transplant recipient. Pharmacotherapy 2006, 26:289-295.

32. Kovarik JM, Hsu CH, McMahon L, et al.: Population pharmacokinetics of everolimus in de novo renal transplant patients: impact of ethnicity and comedications. Clin Pharmacol Ther 2001, 70:247-254.

33. Backman JT, Kivisto KT, Olkkola KT, Neuvonen PJ: The area under the plasma concentration-time curve for oral midazolam is 400 -fold larger during treatment with itraconazole than with rifampicin. Eur J Clin Pharmacol 1998, 54:53-58.

34. Olkkola KT, Ahonen J, Neuvonen PJ: The effects of the systemic antimycotics, itraconazole and fluconazole, on the pharmacokinetics and pharmacodynamics of intravenous and oral midazolam. Anesth Analg 1996, 82:511-516.

35.• Saari TI, Laine K, Leino K, et al.: Effect of voriconazole on the pharmacokinetics and pharmacodynamics of intravenous and oral midazolam. Clin Pharmacol Ther 2006, 79:362-370.

The route of administration of a drug will influence the magnitude of the interaction, which is clearly demonstrated by the comparison of intravenous versus orally administered midazolam.

36. Ahonen J, Olkkola KT, Neuvonen PJ: The effect of the antimycotic itraconazole on the pharmacokinetics and pharmacodynamics of diazepam. Fundam Clin Pharmacol 1996, 10:314-318.

37. Saari TI, Laine K, Bertilsson L, et al.: Voriconazole and fluconazole increase the exposure to oral diazepam. Eur J Clin Pharmacol 2007, 63:941-949.

38. Ariffin H, Omar KZ, Ang EL, Shekhar K: Severe vincristine neurotoxicity with concomitant use of itraconazole. J Paediatr Child Health 2003, 39:638-639.

39.• Bermudez M, Fuster JL, Llinares E, et al.: Itraconazolerelated increased vincristine neurotoxicity: case report and review of literature. J Pediatr Hematol Oncol 2005, 27:389-392.

As with Mantadakis et al. [40•], azoles are more frequently used in the setting of prophylaxis to prevent invasive fungal infections. Yet, these drugs may interfere substantially with anticancer drugs needed for curative therapy.
40. Mantadakis E, Amoiridis G, Kondi A, Kalmanti M: Possible increase of the neurotoxicity of vincristine by the concurrent use of posaconazole in a young adult with leukemia. J Pediatr Hematol Oncol 2007, 29:130.

As with Bermudez et al. [39•], azoles are more frequently used in the setting of prophylaxis to prevent invasive fungal infections. Yet, these drugs may interfere substantially with anticancer drugs needed for curative therapy.

41. Sathiapalan RK, Al-Nasser A, El-Solh H, et al.: Vincristine-itraconazole interaction: cause for increasing concern. J Pediatr Hematol Oncol 2002, 24:591.

42. Marr KA, Leisenring W, Crippa F, et al.: Cyclophosphamide metabolism is affected by azole antifungals. Blood 2004, 103:1557-1559.

43. Bailey EM, Krakovsky DJ, Rybak MJ: The triazole antifungal agents: a review of itraconazole and fluconazole. Pharmacotherapy 1990, 10:146-153.

44. Grant SM, Clissold SP: Itraconazole: a review of its pharmacodynamic and pharmacokinetic properties, and therapeutic use in superficial and systemic mycoses. Drugs 1989, 37:310-344.

45. Ghosal A, Hapangama N, Yuan Y, et al.: Identification of human UDP-glucuronosyltransferase enzyme(s) responsible for the glucuronidation of posaconazole (Noxafil). Drug Metab Dispos 2004, 32:267-271.

46. Purkins L, Wood N, Ghahramani P, et al.: Coadministration of voriconazole and phenytoin: pharmacokinetic interaction, safety, and toleration. Br J Clin Pharmacol 2003, 56(Suppl 1):37-44.

47. Geist MJ, Egerer G, Burhenne J, et al.: Induction of voriconazole metabolism by rifampin in a patient with acute myeloid leukemia: importance of interdisciplinary communication to prevent treatment errors with complex medications. Antimicrob Agents Chemother 2007, 51:3455-3456.

48. Jaruratanasirikul S, Sriwiriyajan S: Effect of rifampicin on the pharmacokinetics of itraconazole in normal volunteers and AIDS patients. Eur J Clin Pharmacol 1998, 54:155-158.

49. Krishna G, Sansone-Parsons A, Kantesaria B: Drug interaction assessment following concomitant administration of posaconazole and phenytoin in healthy men. Curr Med Res Opin 2007, [Epub ahead of print.]

50. Crommentuyn KM, Mulder JW, Sparidans RW, et al.: Drug-drug interaction between itraconazole and the antiretroviral drug lopinavir/ritonavir in an HIV-1-infected patient with disseminated histoplasmosis. Clin Infect Dis 2004, 38:e73-e75.

51. MacKenzie-Wood AR, Whitfeld MJ, Ray JE: Itraconazole and HIV protease inhibitors: an important interaction. Med J Aust 1999, 170:46-47.

52. Liu P, Foster G, Gandelman K, et al.: Steady-state pharmacokinetic and safety profiles of voriconazole and ritonavir in healthy male subjects. Antimicrob Agents Chemother 2007, 51:3617-3626.

As with Liu et al. [54•], patients who are HIV positive are subject to fungal infections. Initiation of antifungal therapy concomitant with highly active antiretroviral therapy may result in a two-way interaction. Both antiretroviral and antifungal therapy should be monitored for clinical failure or toxicity.

53. Mikus G, Schowel V, Drzewinska M, et al.: Potent cytochrome P450 2C19 genotype-related interaction between voriconazole and the cytochrome P450 3A4 inhibitor ritonavir. Clin Pharmacol Ther 2006, 80:126-135.

54. - Liu P, Foster G, Labadie RR, et al.: Pharmacokinetic interaction between voriconazole and efavirenz at steady state in healthy male subjects. J Clin Pharmacol 2007, [Epub ahead of print.]

As with Liu et al. [52•], patients who are HIV positive are subject to fungal infections. Initiation of antifungal therapy concomitant with highly active antiretroviral therapy may result in a two-way interaction. Both antiretroviral and antifungal therapy should be monitored for clinical failure or toxicity. 
55. Purkins L, Wood N, Ghahramani P, et al.: No clinically significant effect of erythromycin or azithromycin on the pharmacokinetics of voriconazole in healthy male volunteers. Br J Clin Pharmacol 2003, 56(Suppl 1):30-36.

56. Ikeda Y, Umemura K, Kondo K, et al.: Pharmacokinetics of voriconazole and cytochrome P450 $2 \mathrm{C} 19$ genetic status. Clin Pharmacol Ther 2004, 75:587-588.

57. Geist MJ, Egerer G, Burhenne J, Mikus G: Safety of voriconazole in a patient with CYP2C9*2/CYP2C9*2 genotype. Antimicrob Agents Chemother 2006, 50:3227-3228.

58. Balian JD, Sukhova N, Harris JW, et al.: The hydroxylation of omeprazole correlates with S-mephenytoin metabolism: a population study. Clin Pharmacol Ther 1995, 57:662-669.

59. Gubbins PO, McConnell SA, Gurley BJ, et al.: Influence of grapefruit juice on the systemic availability of itraconazole oral solution in healthy adult volunteers. Pharmacotherapy 2004, 24:460-467.
60. Pennick GJ, Clark M, Sutton DA, Rinaldi MG: Development and validation of a high-performance liquid chromatography assay for voriconazole. Antimicrob Agents Chemother 2003, 47:2348-2350.

61. Rengelshausen J, Banfield M, Riedel KD, et al.: Opposite effects of short-term and long-term St. John's wort intake on voriconazole pharmacokinetics. Clin Pharmacol Ther 2005, 78:25-33.

62.• Saad AH, DePestel DD, Carver PL: Factors influencing the magnitude and clinical significance of drug interactions between azole antifungals and select immunosuppressants. Pharmacotherapy 2006, 26:1730-1744.

Complete overview of azole interactions with selected immunosuppressant drugs. 